\title{
Entrelacs
}

Cinéma et audiovisuel

$17 \mid 2020$

$N^{\circ} 17$ / Enjeux Audiovisuels du cinéma 360

\section{Être en apesanteur :}

Une approche diégétique en réalité virtuelle

\section{Swann Martinez and Chu-Yin Chen}

\section{CpenEdition}

Journals

Electronic version

URL: http://journals.openedition.org/entrelacs/5918

DOI: 10.4000/entrelacs.5918

ISSN: 2261-5482

Publisher

Éditions Téraèdre

Electronic reference

Swann Martinez and Chu-Yin Chen, «Être en apesanteur : », Entrelacs [Online], 17 | 2020, Online since

01 July 2020, connection on 03 July 2020. URL : http://journals.openedition.org/entrelacs/5918 ;

DOI : https://doi.org/10.4000/entrelacs.5918

This text was automatically generated on 3 July 2020 .

Tous droits réservés 


\title{
Être en apesanteur :
}

\author{
Une approche diégétique en réalité virtuelle
}

\author{
Swann Martinez and Chu-Yin Chen
}

\section{Remerciements}

Nous souhaitons remercier, ici, Kitsou Dubois pour sa direction de projet et le partage de son expérience chorégraphique de l'apesanteur; nos remerciements vont aussi à l'ensemble des membres du projet Le corps infini : la Compagnie Ki Productions, l'Académie Fratellini, l'Université Paris 8 (labo CICM-Musidance / INREV-AIAC), l'ENS Louis-Lumière et l'ENSAD. Nous tenons à remercier particulièrement les artistes circassiennes pour leurs retours si constructifs pour notre réalisation.

Le projet Le corps infini a bénéficié du soutien du Labex Arts-H2H et d'une aide de l'ANR au titre $d u$ Programme Investissements d'Avenir (ANR-10-LABX-80-01). Le projet a également reçu le soutien de La Fondation Carasso.

1 Le dispositif de réalité virtuelle Être en apesanteur est issu du projet Le Corps Infini réalisé dans le cadre du Labex Arts - $\mathrm{H}_{2} \mathrm{H}^{1}$. Ce projet porte sur la question du corps et du mouvement en apesanteur dans l'espace scénique du cirque. S'étant déroulé de 2016 à 2018, le projet de recherche et création Le Corps Infini, réunissant différents partenaires ${ }^{2}$, a mis en place une dynamique d'expérimentation collective au croisement des arts numériques, de l'art audiovisuel, de la danse et du cirque. Il interrogeait la possibilité de créer dans un espace en trois dimensions, réel ou virtuel, une performance artistique suggérant les conditions de l'apesanteur. De nature pluridisciplinaire, il s'agissait d'opérer un basculement de la perception du corps et de l'environnement par l'immersion dans un espace visuel, sonore et virtuel. S'appuyant sur les expériences de Kitsou Dubois (Compagnie Ki Productions) sur le corps et le mouvement en apesanteur, ce projet associait des enseignants, chercheurs et artistes, des étudiants (doctorants, masters et apprentis) de deux écoles (académie Fratellini, ENS Louis Lumière) et de 2 laboratoires de l'université Paris 8 (CICM-Musidance, INREV-AIAC) afin d'initier d'une part une évolution du regard, du point de vue et de l'écoute dans l'art performatif et l'art numérique, et d'autre part, une démarche de partage et de transmission des savoir-faire. 
2 Nourrie par les différentes formes d'expression artistique des partenaires du projet et par le désir de Kitsou Dubois de mettre en scène une performance circassienne s'inspirant de l'absence de gravité, l'équipe INREV a créé le dispositif en Réalité Virtuelle Être en apesanteur. Situés à côté de la scène, les spectateurs étaient invités, après la présentation de la performance, à venir vivre en première personne ${ }^{3}$, la sensation d'être en l'air comme les circassiens. Ils peuvent, pendant un instant, perdre l'impression de pesanteur de par leurs interactions gestuelles relevant d'un contact dialogique reliant un monde réel pesant et un monde virtuel sans gravité.

3 Installé sur une balancelle, le public est invité à explorer un environnement onirique par un toucher virtuel. En effleurant des éléments libérés de leur pesanteur, grâce à des simulations d'objets physiques tels que des matières solides, fluides ou vaporeuses, le spectateur s'approprie son corps virtuel par l'action de ses membres; il entre alors dans un état d'énaction où ses gestes s'incarnent progressivement dans ce monde virtuel sans pesanteur. Douillettement porté par la balancelle, le corps du spectateur se met dans une posture quasi fœtale, comme s'il était soulevé par la main d'une personne, ou bien se trouvait dans le ventre de sa mère, tel un embryon dans sa poche amniotique.

4 En tant que recherche et création, ce dispositif de réalité virtuelle Être en apesanteur nous a amenés à étudier :

5 Dans un premier temps, comment créer un monde donnant l'impression d'une absence de gravité ou suggérer d'un point de vue empathique des perceptions d'apesanteur? En effet, cela différait beaucoup de la création d'un décor, car il s'est surtout agi d'instaurer une diégèse : un cadre chronologique et spatial d'évolution des éléments de cet univers soulignant la spécificité de leurs mouvements propres.

6 Puis, en retour, nous nous sommes demandé comment cette exploration serait vécue par le public, afin de déterminer quels seraient les caractères à même d'induire cette sensation de libre flottement dans un espace éthéré. Par exemple, en quoi des interactions avec des matières fluides et volatiles pourraient-elles venir renforcer cette incarnation dans ce monde, par l'énaction née d'une gestualité de contact dialogique?

7 Nous nous sommes également intéressés à ce qui pourrait prolonger chez le spectateur l'expérience esthétique de la performance, sur le plan d'une plus grande empathie visà-vis des artistes circassiens.

8 Un recueil du vécu des circassiennes et une description complète du processus de création de l'œuvre précéderont notre analyse des mécanismes physiques et virtuels à l'origine de cette réalisation. Puis, une étude des perceptions-représentations et des actions-réactions des spectateurs rendra compte de la manière dont ils interagissent avec l'œuvre et du type d'actes qu'ils adoptent ou improvisent en regard de leurs comportements habituels, ayant ainsi permis une évolution organique de l'œuvre au cours du projet. L'ensemble permettra de valider les résultats de nos recherches et d'améliorer nos créations.

\section{Genèse de l'œuvre}

9 Cette section décrit les conditions initiales du projet ainsi que les enjeux de la première itération de l'œuvre. 
Une particularité du processus de recherche-création à l'origine de cette œuvre réside dans l'environnement de sa naissance. Le plateau expérimental en place alors se tenait dans l'Académie de cirque Fratellini. L'équipe pluridisciplinaire présente était composée de circassiens, cinéastes, compositeurs et de techniciens ayant l'objectif commun de représenter le corps et le mouvement en apesanteur par leur domaine d'expertise artistique. Notre équipe INREV, spécialisée en réalité virtuelle, devrait concevoir une expérience prolongeant celle du spectacle vivant. En écho avec la performance live, nous devions plonger le spectateur dans l'univers des circassiennes de la performance.

\section{Faire appel au vécu des circassiennes}

11 En préalable à toute tentative de transposition de cette expérience en réalité virtuelle, nous nous sommes interrogés sur le contenu sensible que vivaient les deux artistes circassiennes pendant leur performance. Ce recueil du vécu des circassiens s'est fait par la conduite d'entretiens spécifiques ${ }^{4}$ pendant les séances de travail du spectacle. Ces entretiens ont permis aux artistes de se re-positionner sur certains moments clés de leur performance, de les revivre et d'éveiller les micro-phénomènes ainsi que les micro-processus d'une action précise, puis d'en décrire à la fois l'état mental et les sensations corporelles.

Ce qui nous intéressait le plus dans ces évocations était le moment de l'exécution de mouvements corporels destinés à donner l'impression d'une évolution du corps en apesanteur, comme s'il était libéré de la force de la gravité, tout en sachant que dans la réalité terrestre, ces artistes devaient pour y arriver, la combattre doublement. Leurs évocations de ces moments particuliers où l'impression de flotter dans l'espace survenait soudainement, nous ont éclairés sur ce qui pourrait être imaginé d'une gestualité artistique de l'apesanteur, telle que des mouvements très lents, une perte de la verticalité, une dissolution des repères, ainsi que des prises d'appuis différents.

13 L'ambiance lumineuse, en particulier l'alternance contrastée entre la prégnance du noir et la présence de lumières diffuses, sans possibilité de vision précise, suggère une impression de volume qui porte l'être, donnant ainsi un sentiment de légèreté ; le corps a l'air de flotter librement dans un espace infini. Et lorsque la concentration sur les points d'appui du corps s'amenuise, l'attention glisse alors vers d'autres choses, vers le ressenti de ce qui entoure, comme le poids de l'air ou le volume de la lueur. C'est cette perte d'attention visuelle au corps, ce corps rendu invisible, qui permettait aux artistes de mieux être dans la corporalité et le ressenti ${ }^{5}$. Aussi, lors des bascules aériennes sur le cerceau, le corps en torsion s'ouvre, laissant circuler un nouveau volume, et se découvre au sein d'un nouvel espace qu'il sépare et tranche parfois en 2 parties $^{6}$.

Dans le contexte de notre dispositif de Réalité Virtuelle, ces retours nous ont orientés vers l'idée de la balançoire (voir figure 1). En effet, la liberté que gagnent les quatre membres, si les pieds ne reposent plus sur le sol et si les mains ne retiennent pas le corps, pourrait faire survenir un détachement apparent de l'ancrage à la terre, et offrirait une plus grande sensation de l'espace environnant réel ou virtuel. Nous avons également retenu quelques principes clés pour nous guider dans la diégèse du monde virtuel. Tout d'abord, il y a l'ambiance lumineuse et la mobilité du regard sur le haut, le bas et ce qui entoure. Puis lors de l'interaction, la visualisation de la main contribuerait davantage à la présence du ressenti des éléments dans la paume; tandis que la vue des 
pieds encouragerait l'idée de pouvoir agir et flotter, et pourtant le corps restait invisible, non représenté. Enfin, nous avons identifié un besoin de délimiter des espaces, similairement au renversement du corps des circassiennes, par des passages qui s'apparentent à une cassure, à un acte pénétrant.

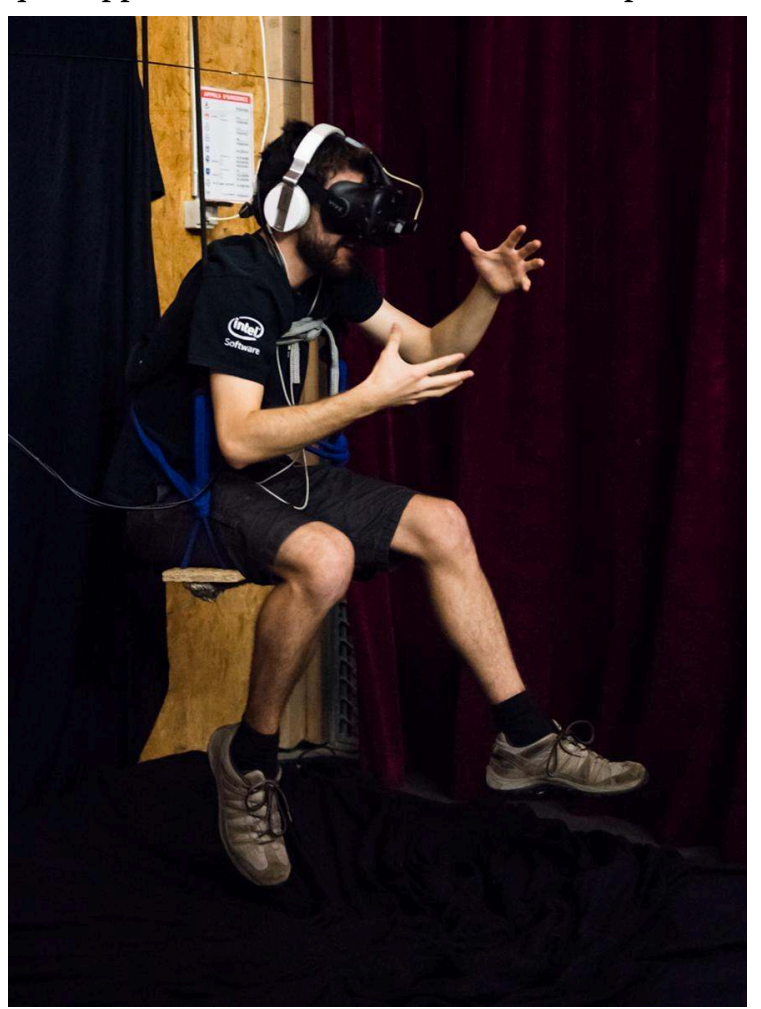

Figure 1: Dispositif initial. 2017

\section{Développement et création du monde virtuel}

15 Parallèlement à la légèreté et à la lenteur d'une gestualité artistique de l'apesanteur, notre fil rouge initial pour tisser cette expérience sensitive en $\mathrm{RV}^{7}$ fut la fluidité de l'eau. En nous appuyant sur le comportement physique de l'eau en apesanteur, nous pourrions guider inconsciemment le spectateur à acquérir une gestuelle proche de celle des corps en apesanteur, telle était notre hypothèse de départ. Cependant, un certain nombre de verrous techniques se sont profilés suite à cette première hypothèse. Comment simuler et rendre des fluides réalistes en temps réel en RV ? Comment créer une atmosphère spatiale convaincante? Comment obtenir un retour audio convaincant des interactions physiques?

16 Nos choix technologiques ont directement découlé de ces problématiques. Le casque de réalité virtuelle permettant de tracker ${ }^{8}$ le plus de membres était alors le HTC Vive. De plus, en y ajoutant un capteur Leap Motion, nous pouvions obtenir une reconstruction très précise des mouvements des doigts dans l'univers virtuel.

En termes de conception du logiciel de l'oeuvre, aucune technologie ne donnait clef en main la possibilité de simuler des fluides en temps réel, il était nécessaire de trouver un moteur de jeux aux sources ouvertes afin de pouvoir étendre ses fonctionnalités. Dans cette optique, le logiciel Unreal Engine 4 était un choix idéal. Pour faire de la simulation de fluides nous avons opté pour FleX, une librairie basée sur les recherches de Miles 
Macklin et al9. Ce framework a pour singularité de paralléliser ses calculs sur processeur graphique, rendant ainsi des interactions temps réels possibles avec la simulation. De plus, une version du logiciel Unreal la mettant partiellement en œuvre était maintenue par NVidia. C'est avec ces différents outils en main que nous avons débuté la phase d'expérimentation-création.

Initialement, notre volonté de transmettre l'expérience de l'apesanteur au public s'est concrétisée autour d'une vision esthétique réaliste du phénomène. Visuellement, nos recherches d'environnements ont naturellement conduit à la conception d'une scène spatiale tangible (voir figure 1) incluant plusieurs points de repère à savoir :

- Une atmosphère volumétrique réaliste ouvrant l'espace.

20 - Différentes planètes lointaines.

21 - Une ville en contrebas rappelant la hauteur.

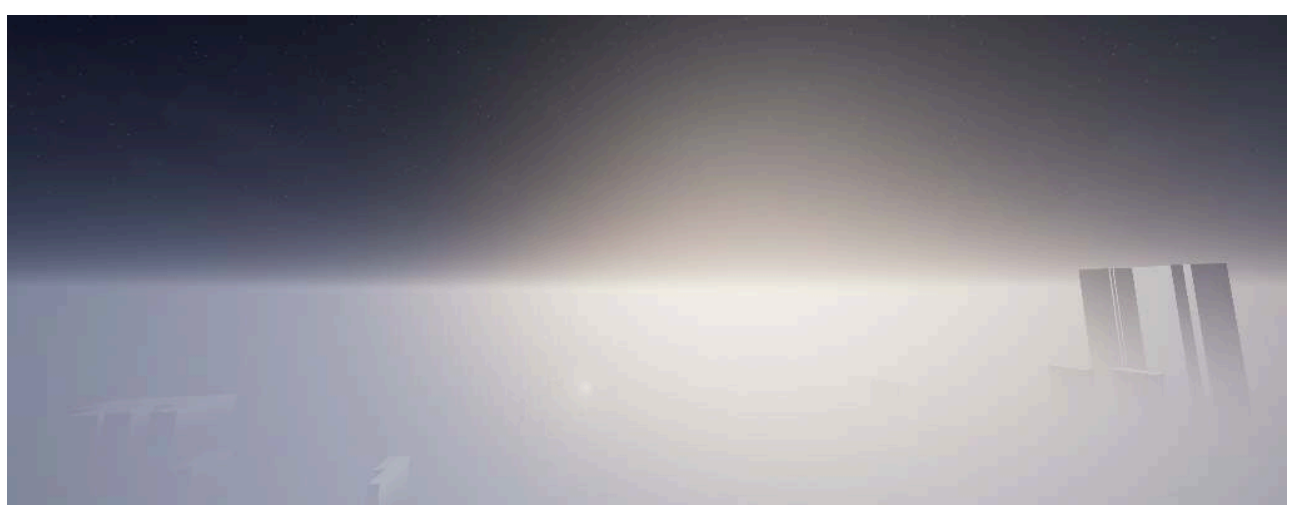

Figure 2 : Environnement spatial initial. 2017

Les mains ont été créées très peu de temps après l'environnement. L'objectif motivant l'utilisation du design présent dans la figure 3 réside dans le concept d'appropriation. Nous avons développé un design générique, mais morphologiquement réaliste. Étant donné que de nombreuses personnes seraient amenées à explorer l'expérience, il était vital de donner à chacun la même perception de ses membres en RV.

Afin d'être en phase avec les différentes équipes du plateau expérimental, nous invitions régulièrement les différents artistes à s'immerger en RV dans cet univers en cours de création. Leurs nombreux retours permettaient d'améliorer et de calibrer l'expérience. Ce processus itératif s'est révélé particulièrement utile lors de la création de la simulation d'eau en apesanteur (voir figure 3). En appliquant les propriétés physiques de l'eau aux paramètres de notre simulation, le fluide en apesanteur était extrêmement fragile: il se fragmentait au moindre contact brutal de la part des spectateurs non-initiés. L'avantage du temps réel dans cette situation fut un gain de temps considérable : les itérations se faisaient instantanément en fonction du ressenti des spectateurs. 


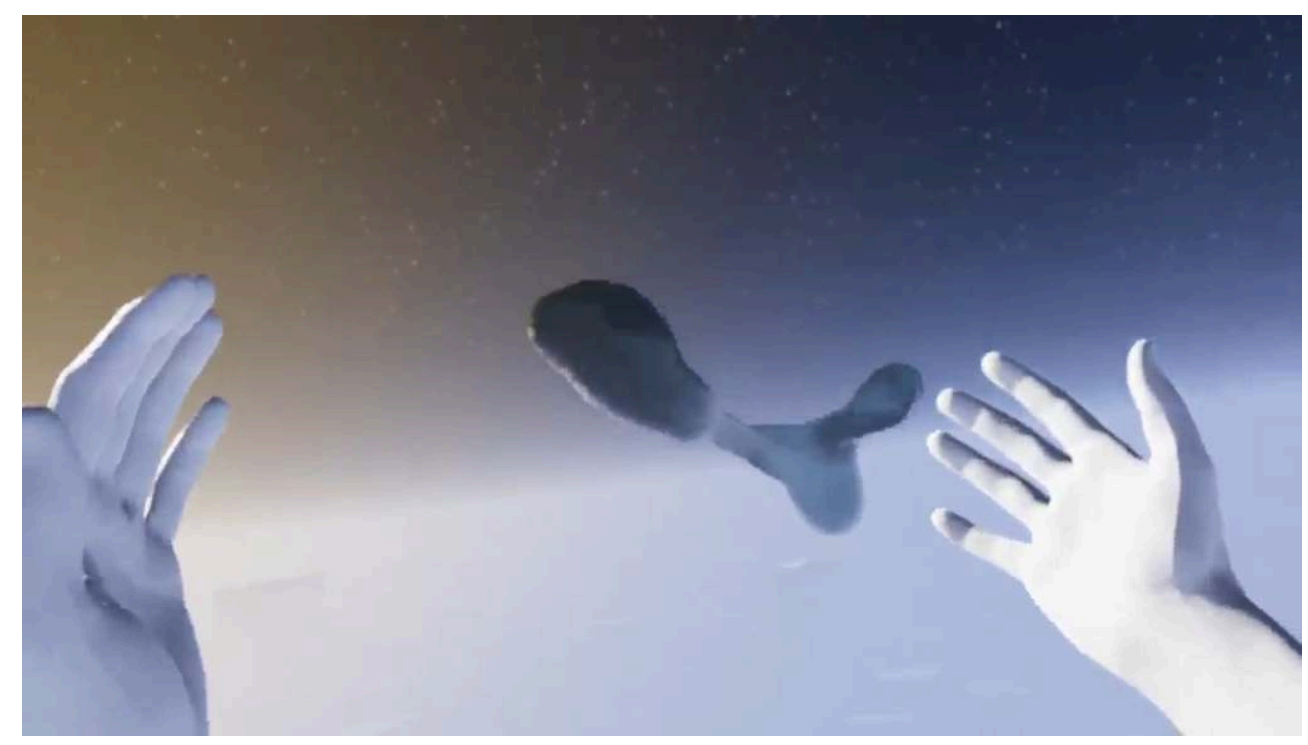

Figure 3 : Rendu initial des mains et du fluide. 2017

$\mathrm{Au}$ terme de 2017, l'expérience invitait le spectateur à interagir avec des fluides en apesanteur dans un décor spatial. Le dispositif physique, constitué d'une balançoire et du casque de réalité virtuelle projetait le spectateur du point de vue empathique d'un trapéziste. Inconsciemment, celui-ci entrait progressivement en phase avec une gestuelle s'apparentant à celle d'un corps en apesanteur. Bien que l'apprentissage de la gestuelle fût une réussite, de nombreux aspects restaient en suspens. Le retour audio des interactions physiques n'était alors que partiel, de plus, certaines personnes mal à l'aise avec la hauteur ont ressenti le vertige. Il s'est avéré que l'expérience ne préparait pas assez le spectateur aux interactions : les éléments diégétiques étaient insuffisants en raison du manque d'indices lui permettant d'appréhender le monde dans lequel il était transporté. En complément, les câbles maintenant les structures de la balançoire limitaient l'amplitude des mouvements possibles. Tous ces éléments se traduisirent par une timidité gestuelle chez le grand public.

La sélection de l'œuvre à Laval Virtual 2018 nous permit de continuer son développement. Il était alors question de produire les éléments diégétiques permettant de soutenir l'apprentissage de la gestuelle et de la dextérité d'un corps en apesanteur.

\section{Approche diégétique en RV}

Cette section fait état de la recherche et de l'approche diégétique qui ont eu lieu au mois de janvier 2018. Les problématiques à l'origine de cette recherche sont multiples: comment désinhiber la gestuelle du sujet ? Par quels moyens induire le sujet à interagir avec des éléments de manière implicite?

\section{Déchirer et trancher}

Comme expliqué précédemment, le cœur de l'expérience réside dans l'interaction physique du spectateur avec les fluides en apesanteur. Un des premiers enjeux consista à amener une progression dans la sensibilité de l'interaction physique. La librairie que nous avons utilisée permettait la simulation de système dynamique en utilisant les lois 
de la physique. Elle nous a permis de simuler un rideau (voir Figure 4). Respectant une gravité terrienne, ce tissu faisait le lien avec la réalité au début et à la fin de l'expérience. En plaçant comme première interaction des tissus, nous faisions appel à quelque chose de connu chez le spectateur. De par sa proximité, cet élément appelle naturellement au contact et induit inconsciemment au toucher. En référence au lever de rideau au théâtre, il était du ressort du spectateur de démarrer l'expérience en écartant ce rideau de ses propres mains.

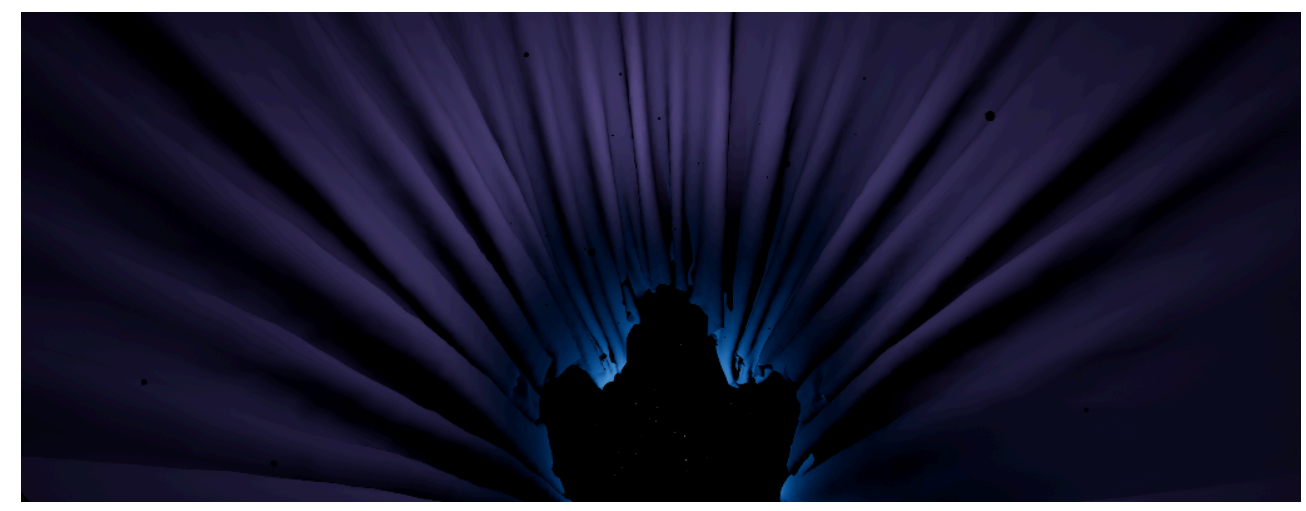

Figure 4 : Simulations de drapés 2018

À ce moment du développement nous avions les scènes d'un commencement, d'un milieu et d'une fin. Cependant, ces fragments diégétiques n'avaient aucune raison d'exister sans connexion. Comment lier narrativement un lieu étroit en pesanteur terrestre avec un environnement vaste en apesanteur?

Il était nécessaire d'imaginer une forme d'ouverture de l'espace, un passage de l'obscurité à la lumière. En suivant le fil rouge de l'interactivité comme élément transitoire, le spectateur devait ouvrir l'espace. Tel un être vivant plongeant dans l'eau, la transition devait plonger l'acteur en apesanteur. Pour concevoir un tel effet, les travaux sur la fragmentation d'objets solides en temps réel de Swann Martinez, basés sur l'article de Mathias Muller et $\mathrm{al}^{10}$ ont été appliqués. En concevant l'espace virtuel initial comme une pièce fermée par quatre murs, il devenait alors possible de simuler une fracture d'objet solide sur ces murs. En procédant de la sorte, nous donnions la possibilité au spectateur-acteur de fragmenter l'environnement sombre pour créer une ouverture vers l'espace (figure 5). Les fragments générés par cette procédure pouvaient ainsi flotter dans l'espace en apesanteur et s'éloigner progressivement afin de ne laisser que le spectateur et l'eau. 


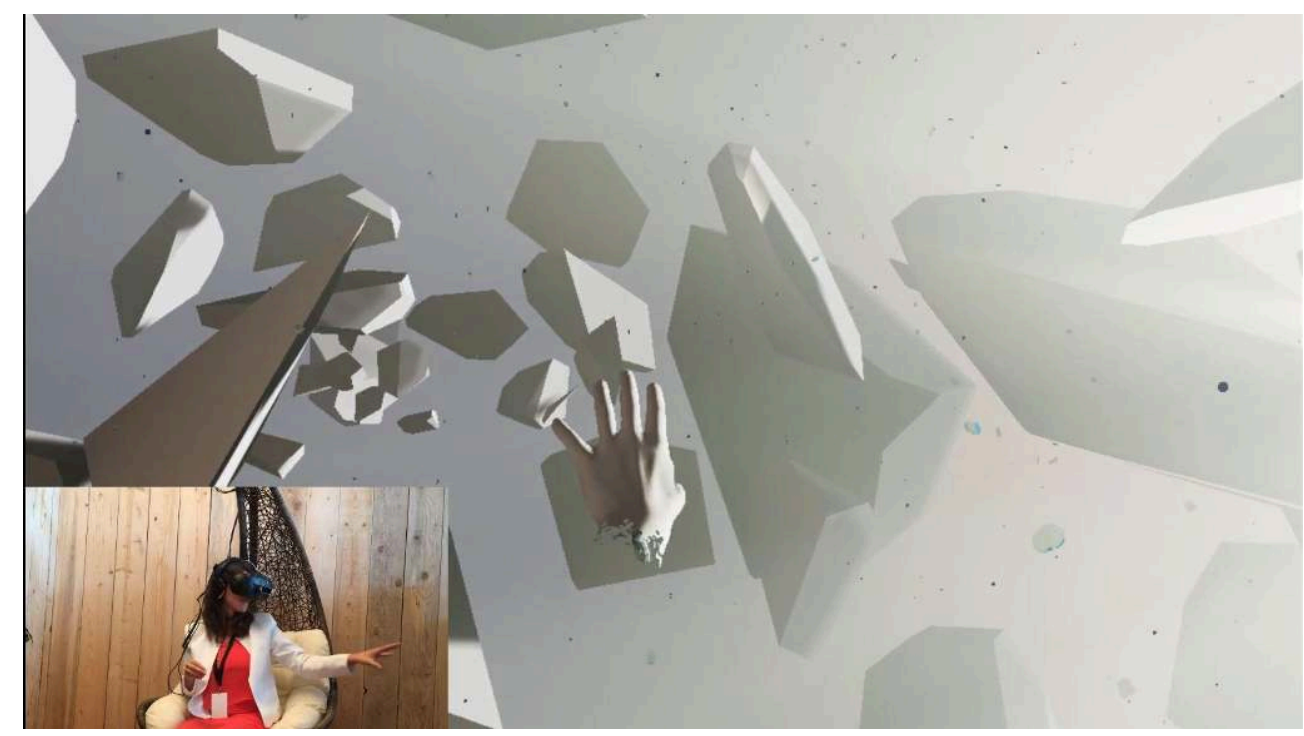

Figure 5 : Simulations de fracture d'objets solides sur les parois de murs

\section{Flotter et effleurer}

Cependant, il n'était pas naturel pour le spectateur de toucher l'obscurité. C'est là que la lumière a joué un rôle crucial : en faisant naître un petit puits de lumière au milieu de l'obscurité, nous guidions la curiosité du spectateur vers un point précis (voir Figure 6). De ce tunnel émane une lumière intense et volumétrique. La réaction naturelle induite par cette vive source lumineuse consiste à essayer de la toucher. Au contact de la main, le mur se fracture, tandis que l'espace sombre et vide se remplit de lumière. Une fois dans l'espace, les interactions avec l'eau commencent. En exagérant l'effet volumétrique de la lumière, il nous est apparu une vision plus abstraite de la scène spatiale. Nous avons retiré les repères au sol afin de focaliser l'attention du spectateur sur les gouttes d'eau. En perdant ainsi tous ses repères, un utilisateur sujet au vertige ne pourrait plus le ressentir.

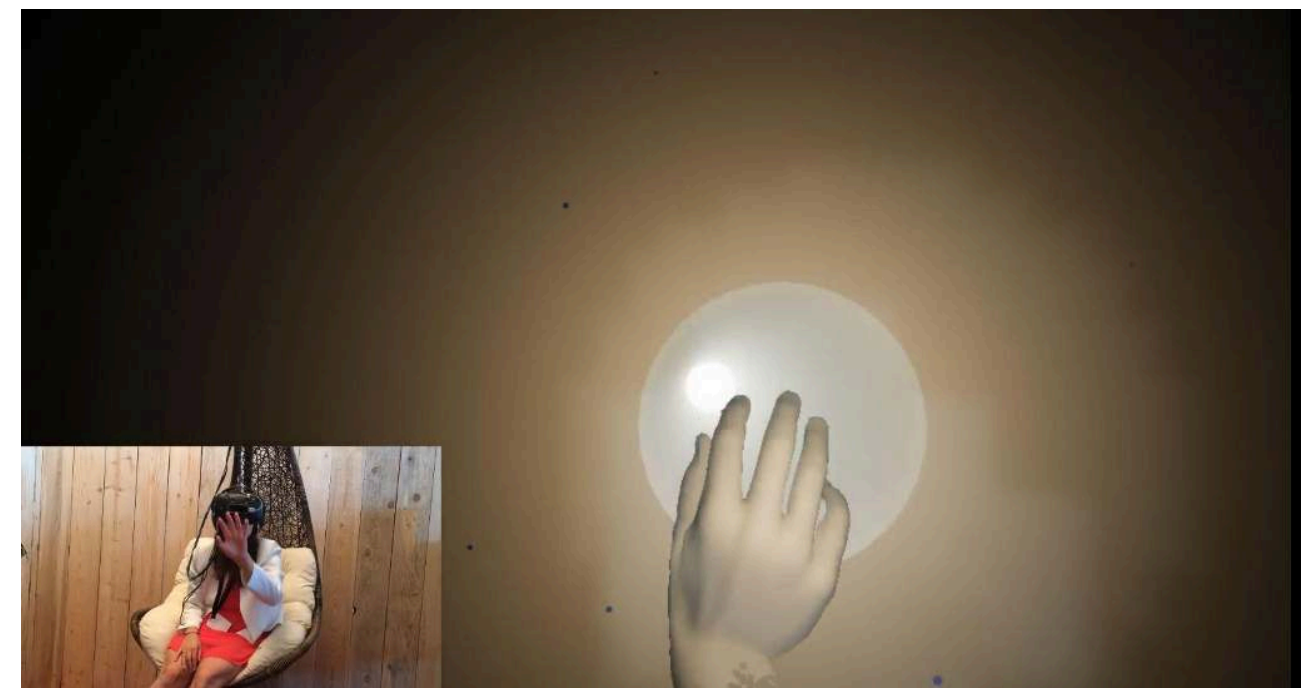

Figure 6 :Ouverture du tunnel lumineux 
Après avoir élaboré cette descente vers la pesanteur venait la question du retour à la réalité. Comment faire revenir progressivement l'univers à pesanteur terrestre?

Dans la même idée que la fragmentation de l'espace obscur, une fragmentation de l'environnement spatial a été utilisée. Pour la concevoir, le rendu de la vue 3D a été projeté sur la surface d'un cube qui se fragmente au toucher du spectateur. Au moment de la fragmentation, l'environnement change et le cube se fracture. Du point de vue de l'acteur en réalité virtuelle, des fragments de l'espace lumineux se diffusent dans l'obscurité (voir figure 7). Après une courte durée, ces derniers se dissipent et ne laissent place qu'au vide. Enfin, le rideau final s'abat autour du spectateur, bouclant ainsi le cycle de l'expérience.
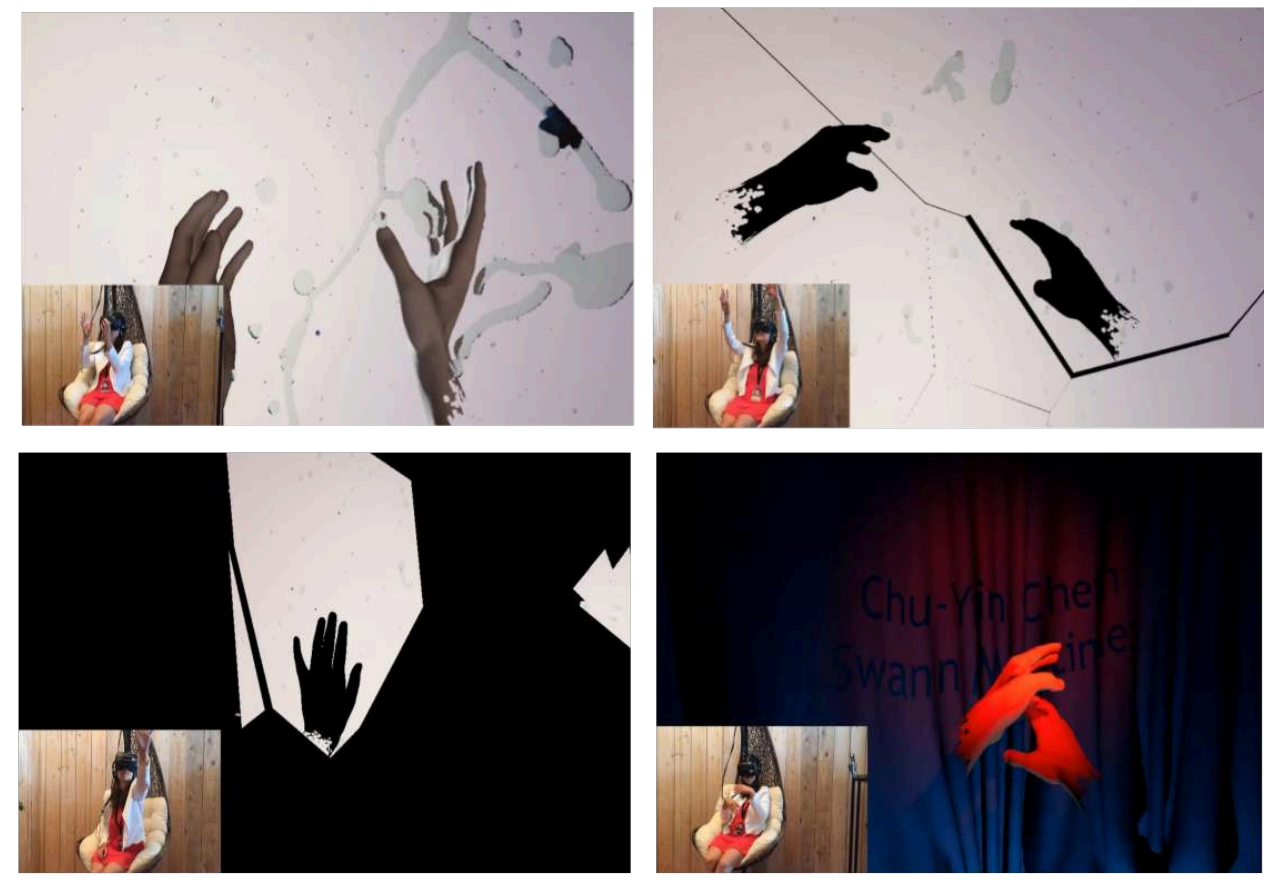

Figure 7 : Évolution de l'environnement au cours du temps

\section{Vecteurs d'immersion}

Les éléments diégétiques précédemment énoncés ont établi une progression dans le changement de pesanteur. Le graphique ci-dessous illustre cette évolution au cours du temps de l'expérience. Cette variation quasi-linéaire immerge le spectateur et le fait ressortir de l'immersion avec douceur en respectant un temps d'adaptation propre à chaque sens. Plus globalement, les transitions construites à partir d'éléments interactifs physiques jouent un rôle crucial de «synchronisation » entre le spectateur réel et son interface virtuelle. En entrant dans l'univers, l'utilisateur doit s'approprier ses mains virtuelles afin de bénéficier d'une expérience complète de l'œuvre. S'il conserve dans son esprit, une dissociation entre ses membres virtuels et ses membres réels, son sentiment de présence serait grandement réduit. À l'inverse, il est extrêmement important que ce dernier récupère ses membres réels progressivement. 
Évolution de la pesanteur au cours du temps

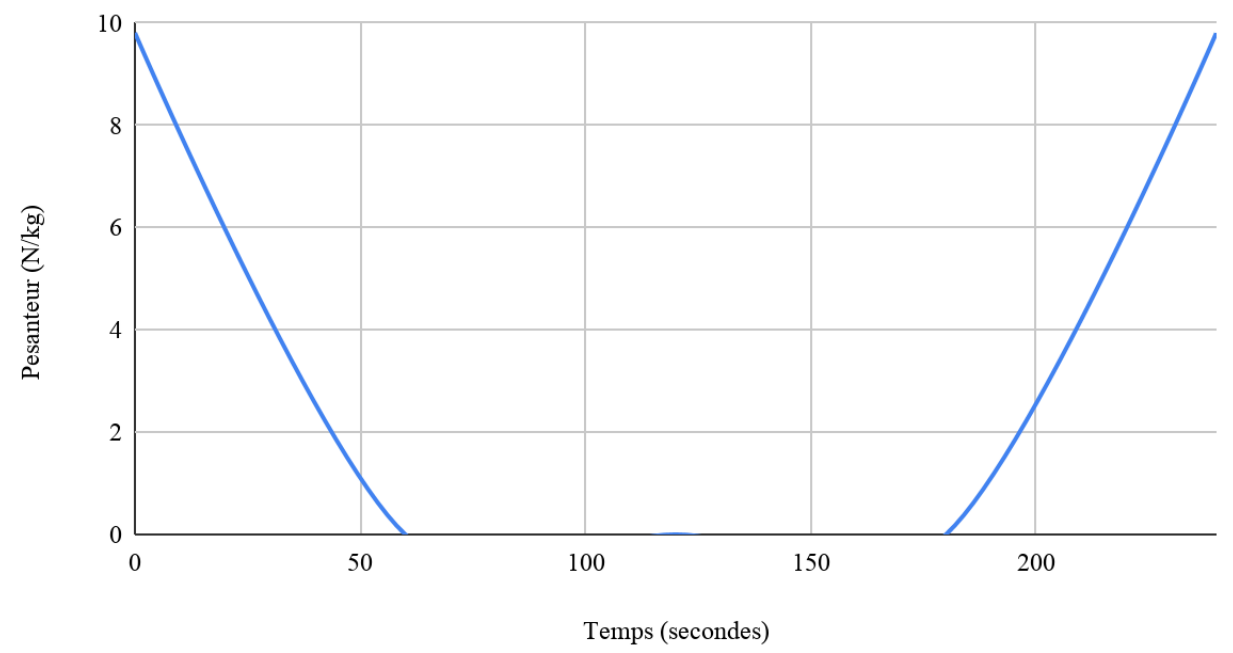

Figure 8 : Évolution de la pesanteur au cours du temps

Dans la version antérieure ne comportant pas de trame narrative, la fin coupait brutalement le spectateur de l'univers virtuel ce qui entraînait chez certains sujets très "synchronisés » une sensation de dissociation avec leurs propres membres. Cet effet indésirable et potentiellement dangereux ne se produisait plus avec cette narration physique.

Après avoir fait découvrir Être en apesanteur à un public très nombreux durant Laval Virtual, beaucoup de retours nourrirent nos réflexions autour de l'évolution de l'œuvre. Malgré la nouvelle diégèse en place, les câbles de la balançoire restreignaient l'amplitude des mouvements possibles et les pieds n'étaient pas supportés en RV. Ces deux éléments constituèrent nos principaux axes de recherches dans les mois qui suivirent.

\section{Vers une libération gestuelle}

Dans cette section, nous aborderons la dernière phase de développement de l'œuvre $\hat{E}$ tre en apesanteur. Dans un premier temps, l'exploration d'une nouvelle plateforme physique sera décrite. La création ainsi que l'étude de la reproduction des membres inférieurs (pieds) dans l'expérience feront l'objet de la seconde partie du chapitre.

\section{De la balançoire à la balancelle}

L'acceptation de l'œuvre au festival Bains Numériques 2018 - biennale internationale des arts numériques d'Enghien-les-Bains débloqua le temps et les moyens nécessaires pour la remise en question de l'installation physique. La problématique étudiée était la suivante : comment abolir les limites de l'amplitude gestuelle physique liée au dispositif physique? 
Pour rappel, la balançoire utilisée comme support contraignait la posture et les gestes de plusieurs manières :

- Les cordes de soutien limitaient des mouvements latéraux des bras et bloquaient la rotation verticale.

- Le cordon de sécurité verrouillait l'utilisateur dans une posture verticale rappelant la gravité.

- La reconnaissance des mains était affectée par l'occlusion des cordes devant la caméra.

Parmi les solutions envisagées, la plus intéressante demeura la balancelle. La figure 8 illustre la mise en situation de la balancelle dans le cadre de l'œuvre. On constate que l'amplitude latérale est totale, de plus la posture légèrement inclinée casse la verticalité de la pesanteur et augmente la sensation de flottement. Enfin, le maintien du support de la balancelle sur un axe unique apporte une liberté de rotation verticale: le spectateur peut tourner à $360^{\circ}$. La balancelle permit également d'éliminer les cordes du champ visuel induisant ainsi une meilleure performance de la captation des mains. Ce nouveau dispositif physique fut éprouvé par plusieurs centaines de personnes durant le festival Bains Numériques 2018. Il s'est avéré d'après les retours de ces derniers que l'expérience leur parut apaisante, flottante. Ces deux qualificatifs faisaient l'unanimité dans les verbatim des spectateurs au sortir de l'expérience. Cependant, au fur et à mesure des passages sur la nouvelle balancelle, un élément inédit ressortait dans la « danse gestuelle » des spectateurs : leurs pieds suivaient une transe similaire à leurs membres supérieurs. L'idée d'inclure les pieds dans les membres virtuels émergea alors, en virtualisant ces deux extrémités nous pourrions amplifier la gestuelle du spectateur.

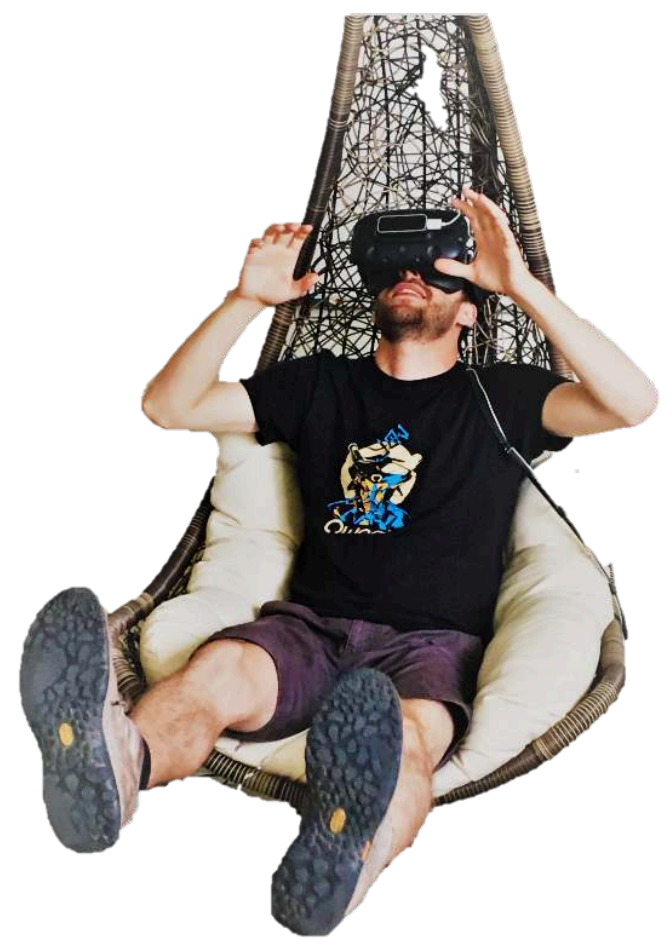

Figure 8 : Utilisation de la balancelle comme support physique. 
Pour commencer à représenter les pieds en réalité virtuelle, une logique similaire à celle utilisée avec les mains fut sollicitée. Une contrainte notable résidait dans l'aspect invasif des capteurs choisis pour la captation à savoir les équipements Vive Trackers. Pour fonctionner correctement, une fixation robuste du capteur à l'objet ciblé pour le tracking était nécessaire. Comment fixer solidement les trackers aux pieds des utilisateurs sans rendre le processus trop invasif afin de ne pas briser l'appairage corps réel/virtuel?

La solution émergeant au sortir de ce questionnement prit la forme d'une paire de chaussons large sur lesquels nous avons consolidé les trackers. Ainsi, pour entrer dans l'expérience, les spectateurs-acteurs devraient chausser ces "souliers augmentés", enfiler le corps virtuel. Ce choix impacta directement les décisions artistiques relatives à la représentation virtuelle des pieds. Toujours dans un souci d'appropriation optimale du corps virtuel, le visuel des pieds de synthèse devait s'approcher au maximum des pieds réels. Les « souliers augmentés » furent donc modélisés en 3D à l'échelle avec un niveau de détail suffisant à leur acceptation naturelle par le spectateur. D'un point de vue empathique, ces derniers apportèrent énormément du fait qu'ils incarnent la représentation exacte d'un élément connu du spectateur transposée dans le monde virtuel. C'est un point d'accroche qui, bien utilisé, pouvait aboutir à une augmentation de la présence virtuelle de l'utilisateur.

\section{Le rôle de la poche embryonnaire}

La virtualisation des membres inférieurs fut rapide à déployer techniquement, mais un problème se posa dès les premières expérimentations avec les membres du laboratoire : bien qu'ayant accès à cette nouvelle extension corporelle virtuelle, les spectateursacteurs ne l'utilisaient pas. L'expérience telle qu'elle était conçue alors ne guidait pas le spectateur dans l'appréhension de ses pieds, mais uniquement dans celle des mains. Nous nous sommes donc interrogés sur la manière d'amener le spectateur à s'approprier simultanément ces extensions virtuelles de ses pieds et de ses mains.

De toute évidence, une nouvelle phase introductive était nécessaire pour faire naître le spectateur entier dans son corps virtuel. Rapidement, une vision du développement embryonnaire émergea de la notion de naissance. L'idée était de mettre le spectateur dans le placenta, de lui faire incarner un embryon humain. Les parois de la poche embryonnaire devaient avoir des propriétés physiques réalistes: se déformer au toucher du spectateur à la manière d'un corps souple. Pour développer cette nouvelle phase diégétique, nous nous sommes appuyés sur les mêmes technologies de simulation physique que pour les fluides. De ce fait, la création de la physique de la poche fut rapide. La difficulté résida dans la recherche de son apparence visuelle. Le chemin de sa création suivit celui du paysage spatial décrit dans le chapitre Développement et création du monde virtuel de cet article.

Nous commençâmes par une approche réaliste avec un rendu de la paroi très organique, sanguin, sombre. Mais la confrontation de cette vision avec le public nous fit prendre conscience de points négatifs non négligeables dans son appréhension par le spectateur :

- La paroi réaliste induit un sentiment de répulsion, de dégoût.

- La faible luminosité produit un fort sentiment de claustrophobie. 
Tout comme pour le paysage, notre seconde approche nous guida vers l'abstraction. Pour gagner la confiance du spectateur, nous devions rendre cet environnement embryonnaire chaleureux, apaisant et vivant. Nous rendîmes donc les parois semitransparentes pour laisser passer une lumière diffuse, chaleureuse et apaisante de par ses pulsations semblables à celles du rythme lent d'un cœur en situation de repos. La géométrie de la poche ne changea pas, morphologiquement fidèle à une poche embryonnaire. Cette seconde représentation fit l'unanimité chez notre public. Une nouvelle transition fut développée : pour achever la naissance, la membrane s'ouvrait et se dissolvait pour laisser la place à l'immensité spatiale remplie de fragments s'éloignant.

Avec cette "naissance ", le public voyait son corps entièrement projeté dans l'univers. Le retour à l'origine de l'être a permis de libérer tous les membres, d'ouvrir la porte à une gestuelle nouvelle. Les résultats furent éloquents, d'un point de vue externe, la gestuelle des spectateurs-acteurs exprimait le ballet d'une naissance spatiale rendant ainsi l'expérience captivante pour le public spectateur (en file d'attente).

\section{Conclusion}

Le contexte riche et unique de la performance du Corps Infini nourrit directement la création de l'œuvre. En prolongeant l'expérience des spectateurs vers le point de vue empathique des circassiennes, l'installation de réalité virtuelle Être en apesanteur donne une interprétation riche et onirique augmentant la performance des circassiennes.

Le cheminement créatif de l'œuvre décrit dans cet article est la synthèse des expérimentations de recherche-création menées entre 2017 et 2018 dont découle Être en apesanteur. Un exposé de l'approche innovante utilisée lors de la conception de l'œuvre s'appuyant sur les ressentis qui transparaissent dans les entretiens d'Explicitation des artistes circassiennes a mis en lumière les différents mécanismes diégétiques utilisés pour guider le spectateur-acteur dans l'appréhension d'un univers en apesanteur. Au fur et à mesure de sa progression à travers un environnement immersif interactif en perpétuel changement, il apprend à s'approprier son corps virtuel. Induit par le dispositif de la balancelle, le flottement apaisant ressenti provoque alors un laisseraller permettant au spectateur-acteur de s'ouvrir à une danse, une gestuelle lente et délicate, quelque peu inconsciente, avec les fluides avec lesquels il interagit. Le niveau de disponibilité du sujet constitue le principal facteur variant de la profondeur de cette transe gestuelle.

\section{BIBLIOGRAPHY}

Références 
Depraz Natalie ; «Qu'est-ce qu'une phénoménologie en première personne ? Premiers pas vers une lecture et une écriture expérientielles (LEE)»; dans Première, deuxième, troisième personne ; Natalie Depraz (dir.) ; Zeta books ; Bucarest ; 2014 ; pp. 118-147.

Dubois Kitsou ; Application des techniques de la danse à l'entraînement du vol en apesanteur : une danseuse en apesanteur ; Thèse de Doctorat, Université Paris 8, 1999. ( $\mathrm{N}^{\circ} 1999$ PA081582).

FuchsPhilippe ; Théorie de la réalité virtuelle : les véritables usages ; Mines Paristech : PSL ; Paris ; 2018.

Guez Judith ; Illusions entre le réel et le virtuel (IRV) comme nouvelles formes artistiques : présence et émerveillement ; Thèse de Doctorat, Université Paris 8, 2015. http://www.theses.fr/2015PA080109.

Macklin Miles, Müller Matthias, Chentanez Nuttapong et Kim Tae-Yong; « Unified Particle Physics for Real-Time Applications »; dans ACM Transactions on Graphics (TOG) Vol. 33, no 4 (July 2014), http://doi.acm.org/10.1145/2601097.2601152.

Missolz Jérôme, Dubois Kitsou ; Kitsou Dubois, une danseuse en apesanteur ; Reportage Vidéo. DVD (24mn) ; Editeur: Centre national de la cinématographie ; Paris ; 2009.

Müller Matthias, Chentanez Nuttapong, et Kim Tae-Yong; « Real Time Dynamic Fracture with Volumetric Approximate Convex Decompositions. »; dans ACM Transactions on Graphics (TOG) Vol. 32 (July 1, 2013), https://doi.org/10.1145/2461912.2461934.Vermersch Pierre ; L'entretien d'explicitation (8e édition augmentée) ; ESF éditeur ; 2014.

\section{APPENDIXES}

\section{Annexe}

Extrait des entretiens d'élicitation avec les circassiennes pendant les séances de travail à l'académie Fratellini, le 13 oct. 2017 de 16 h à 18 h : Lorette S. nous a fait part de la sensation de l'espace infini et de l'influence du regard :

[il y a juste une main qui tient, tout le reste - mon corps - est en train de flotter, c'est vraiment la sensation de flotter et d'avoir de l'espace, et d'avoir l'imaginaire d'être dans l'espace....]

[J'aime bien penser que les mains et les pieds touchent l'air, que l'air est une matière plus que rien, et que je bouge, plongé dans une matière qui me ralentit un peu, cela me fait prendre conscience de tout l'espace autour.]

[Lorsque ma tête n'est plus en train de regarder mon bras, et comme je vois du noir tout autour de moi et des lumières un peu, mais sans voir d'objets précis, cela me donne vraiment l' impression d'être dans l'espace, qu'on pourrait dire l'espace infini ... Et quand je ne vois pas trop la distance, c'est là que j'oublie que j'ai un poids.]

[Le regard a beaucoup d'influence sur mon corps, sur moi; je devrais essayer de faire la performance avec les yeux fermés, dans le noir ..., si je regarde moi-même (mon corps), je suis plus centré sur moi et j'arrive moins à être dans la corporalité (dans le ressenti du corps).] Lauranne W. nous a fait part du ressenti corporel et spatial lors d'un basculement :

[À ce moment-là, j'ai le corps en torsion, avec une partie de moi qui voit l'espace en bas, et l'autre partie l'espace en haut; cela me donne l'impression de passer d'une parallèle au sol à une parallèle au ciel, avec un espace compact au-dessus. Je me sens comme une ligne d'horizon qui sépare deux volumes, un au-dessus et un au-dessous.] 
[Lorsque je me retourne, je ressens le volume de l'espace, poids de l'air ; en fait, je sens moins bien l'espace avec le dos, je le fais mieux avec la paume, le visage.]

[Le mouvement du corps, la torsion du corps fait ouvrir, fait rencontrer un autre espace, on le sent, car on le tranche! C'est une rencontre avec tout son corps.

J'ouvre, l'air rentre, un volume rentre ici, et peut recevoir... est-ce le même espace? Oui, la sensation est la même, mais j'ai changé d'espace, c'est un espace différent, car avant je voyais le bas et maintenant le haut.]

[Cela me donne la conscience de l'espace qui donne un poids selon ma position et la manière dont je reçois l'air et l'espace. Cela passe par mon corps comme si la peau réagissait à un contact concret, comme une texture qui arrive d'un coup sur elle, sentir le volume éveille la peau, je sens que je porte un espace qui se pose sur moi.]

[Quand je suis vers le haut, mon corps devient plus grand, comme une surface qui porte tout l' espace; j'associe cela à un état du corps plus pétillant, avec plus de l'énergie; quand je suis posé (vers en bas) sur l'espace, mon corps me semble plus dense ; posé à l'inverse (vers en haut) c'est l'espace qui a le poids et mon corps qui est plus léger, est-ce que je porte l'espace ou je suis collée àl'espace?

L'énergie monte vers le haut et se mélange à l'espace. Je sens que cela circule par le haut, et occupe tout l'espace en haut, cela devient l'espace! Comme j'ai conscientisé cet espace, mon énergie peut circuler dedans; mon corps est étiré et mes sensations sont quelque chose d'étiré qui diffuse.]

\section{NOTES}

1. Laboratoire d'Excellence Arts et Médiation Humaines (Arts-H2H), http://www.labex-artsh2h.fr/

2. Les Partenaires du projet étaient: ENS Louis-Lumière, Compagnie Ki Productions, Académie Fratellini, Université Paris 8 (labo CICM-Musidance / INREV-AIAC), l'ENSAD.

3. Ainsi que le définit Natalie Depraz dans son article «Qu'est-ce qu'une phénoménologie en première personne? Premiers pas vers une lecture et une écriture expérientielle (LEE)»; dans Première, deuxième, troisième personne; Natalie Depraz (dir.) ; Zeta books ; Bucarest ; 2014 ; pp. 118-147.

4. Il s'agit d'entretiens d'élicitation pratiqués au sein de l'équipe INREV, favorisant la conscientisation des pratiques artistiques et le recueil de données qualitatives et singulières. (Cf. https://inrev.univ-paris8.fr/spip.php?article1543). Cette méthode est dérivée de celle que Pierre Vermersch décrit dans son livre, L'entretien d'explicitation (8e édition augmentée) ; ESF éditeur ; 2014.

5. Voir l'extrait de l'entretien de Lorette en Annexe.

6. Voir l'extrait de l'entretien de Lauranne en Annexe.

7. Réalité Virtuelle

8. Le néologisme 'Tracker' est issu de l'anglais to track. Il est utilisé en image de synthèse dans le sens d'enregistrer le mouvement d'un élément.

9. Macklin Miles, Müller Matthias, Chentanez Nuttapong et Kim Tae-Yong; « Unified Particle Physics for Real-Time Applications »; dans ACM Transactions on Graphics (TOG) Vol. 33, n 4, Juillet 2014, http://doi.acm.org/10.1145/2601097.2601152 
10. Müller Matthias, Chentanez Nuttapong, et Kim Tae-Yong; « Real Time Dynamic Fracture with Volumetric Approximate Convex Decompositions. "; dans ACM Transactions on Graphics (TOG) Vol. 32, 1 Juillet 2013, https://doi.org/10.1145/2461912.2461934

\section{ABSTRACTS}

\section{Abstract}

The artistic installation Being in weightlessness studied in this article is born from the Labex Art \& $\mathrm{H} 2 \mathrm{H}$ Infinite Body project which is gathering multiple artistic representations of bodies in suspension in the circus scenic space. This installation is both a research-creation process and a result of experimentations produced around the project. It led us to question diegetic characteristics of a zero-gravity virtual world and how interactions with fluids and volatile materials could improve feelings of incarnation in this world.

Being in weightlessness invited the public to experience weightless feelings from the aerial viewpoint of the Circassians during the Infinite Body performance. By exploring an environment without gravity, the spectator enters a state of enaction while his gestures are gradually embodied in his virtual body. Comfortably suspended in a spherical swing, he puts himself in an almost fetal position as if it was supported by an invisible hand, like an embryo in its amniotic sac.

Résumé

Le dispositif de réalité virtuelle Être en apesanteur faisant l'objet de cet article est issu du projet du Labex Arts-H2H Le Corps Infini qui réunissait différentes formes d'expression artistique du corps en apesanteur dans l'espace scénique du cirque. Cette installation est à la fois un processus de recherche création et un des fruits des expérimentations réalisées autour du projet. Elle nous a conduit à s'interroger sur les caractéristiques diégétiques d'un monde virtuel suggérant des perceptions d'apesanteur, et en quoi des interactions avec des matières fluides et volatiles pourraient venir renforcer cette sensation d'incarnation dans ce monde.

Lors de la performance Le Corps Infini, le monde virtuel Etre en apesanteur invitait le public à vivre le point de vue aérien des circassiennes, et une sensation de gravité altérée. En explorant un environnement onirique sans pesanteur, le spectateur entre dans un état d'énaction lorsque ses gestes s'incarnent progressivement dans son corps virtuel. Porté par une balancelle, il se met dans une posture quasi fœtale, comme s'il était soutenu par la main d'une personne, tel un embryon dans sa poche amniotique.

\section{AUTHORS}

\section{SWANN MARTINEZ}

doctorant en Cifre chez Cube Creative Productions, Equipe Image Numérique et Réalité Virtuelle (INREV) du laboratoire Arts des Images - Art Contemporain, université Paris 8. Sujet de thèse : Processus de création temps réel pour le cinéma d'animation. 


\section{CHU-YIN CHEN}

Artiste et Professeur, Equipe Image Numérique et Réalité Virtuelle (INREV) du laboratoire Arts des Images - Art Contemporain, université Paris 8 . Thèmes de recherche : Vie artificielle et Systèmes complexes pour l'Art numérique énactif, Elicitation et Pleine conscience comme moyen et processus de recherche-création. 